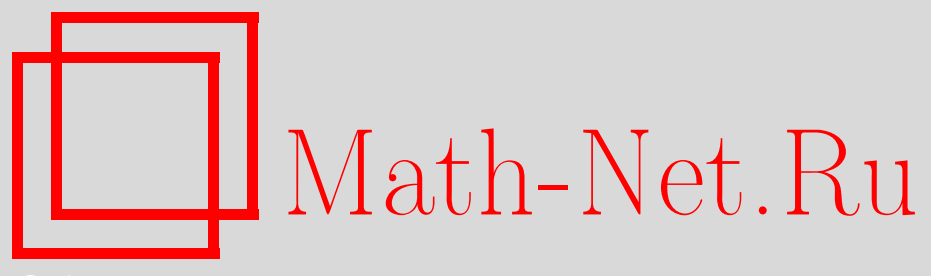

Х. А. Ахадкулов, О некоторых гомеоморфизмах окружности с особенностями типа излома, УМН, 2006, том 61, выпуск 5, 183-184

DOI: https://doi.org/10.4213/rm4469

Использование Общероссийского математического портала Math-Net.Ru подразумевает, что вы прочитали и согласны с пользовательским соглашением http://www.mathnet.ru/rus/agreement

Параметры загрузки:

IP : 54.224 .60 .19

26 апреля 2023 г., 12:30:43

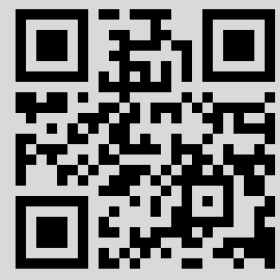




\section{О некоторых гомеоморфизмах окружности с особенностями типа излома}

\section{Х. А. Ахадкулов}

В настоящей работе изучается гомеоморфизмы окружности $S^{1}=[0,1)$ с изломами, т.е. отображения, гладкие всюду за исключением нескольких особых точек, где происходит скачок первой производной. При этом рассматривается случай, когда вторая производная определена лишь почти наверное и принадлежит классу $L_{p}, p>1$. Оказывается, что значительная часть теории, развитой для гладких диффеоморфизмов, переносится на этот случай.

Рассмотрим сохраняющий ориентацию гомеоморфизм окружности $T_{f} x=\{f(x)\}$, $x \in S^{1}$, где скобки $\{\cdot\}$ обозначают дробную часть числа, а функция $f(x)$, определяющая гомеоморфизм $T_{f}$, обладает следующими свойствами: (а) $0 \leqslant f(0)<1$; (b) $f(x+1)=f(x)+1$ для любого $x \in \mathbb{R}^{1}$; (c) гомеоморфизм $T_{f}$ в точках $z_{j}, j=\overline{0, m}$, имеет изломы, т.е. существуют положительные односторонние производные $f^{\prime}\left(z_{j} \pm 0\right)$, $j=\overline{0, m}$, и $\frac{f^{\prime}\left(z_{j}-0\right)}{f^{\prime}\left(z_{j}+0\right)}=c\left(z_{j}\right) \neq 1, j=\overline{0, m}$; (d) $f^{\prime}(x)$ абсолютная непрерывная функция на каждой связной компоненте множества $S^{1} \backslash\left\{z_{j}, j=\overline{0, m}\right\}, f^{\prime}(x) \geqslant$ const $>0$ $\forall x \in S^{1} \backslash\left\{z_{j}, j=\overline{0, m}\right\}$; (е) $f^{\prime \prime}(x) \in L_{p}\left(S^{1}, d x\right)$ при некотором $p>1$.

Смысл условий (а)-(е) состоит в том, что функция $f(x)$ определяет кусочно гладкий гомеоморфизм окружности, имеющий $(m+1)$ точек излома.

Хорошо известно (см. [1]), что для любого $x \in \mathbb{R}^{1}$ существует предел $\lim _{n \rightarrow \infty} \frac{f^{n}(x)}{n}$ $=\rho(f)$, где $f^{n}(x)$ обозначает $n$-ю итерацию функции $f$. Число $\rho(f)$, называемое числом вращения, не зависит от выбора $x$ и является важнейшей характеристикой гомеоморфизма $T_{f}$.

Пусть число вращения $\rho(f)$ иррационально. Тогда отображение $T_{f}$ является строго эргодическим, т.е. обладает единственной вероятностной инвариантной мерой $\mu[1]$. Отсюда, в частности, следует, что $T_{f}$ эргодично относительно инвариантной меры $\mu$.

Обозначим $\lambda$ меру Лебега на окружности $S^{1}$.

ОПРедЕЛЕниЕ 1 . Отображение $T_{f}$ называется эргодическим относительно меры Лебега, если для любого инвариантного множества $A$ его мера $\lambda(A)$ равна 0 или 1.

Следующий результат обобщает теорему Катка, доказавшего эргодичность относительно меры Лебега для диффеоморфизмов класса $C^{2}$ (см. [1]).

Теорема 1. Пусть функиия $f(x)$, определяющая гомеоморфизм $T_{f}$, удовлетворяет условиям (a)-(f) и число вращения $\rho=\rho(f)$ иррачионально. Тогда $T_{f}$ эргодично относительно меры Лебега $\lambda$.

Из теоремы 1 легко вытекает, что инвариантная мера $\mu$ или абсолютно непрерывна, или сингулярна относительно меры Лебега $\lambda$, т.е. существует измеримое подмножество $A$ окружности $S^{1}$ такое, что $\mu(A)=1$ и $\lambda(A)=0$.

Обозначим через $T_{\rho}$ поворот окружности на угол $\rho$. Теорема Данжуа утверждает [2], что если $f \in C^{1}\left(\mathbb{R}^{1}\right)$ и $\operatorname{var}_{S^{1}} \ln f^{\prime}(x)<\infty$, то в случае иррационального числа вращения $\rho$ диффеоморфизм $T_{f}$ и линейный поворот $T_{\rho}$ топологически эквивалентны, т.е. существует сохраняющий ориентацию гомеоморфизм окружности $T_{\varphi}$ такой, что $T_{\varphi} \circ T_{f}=T_{\rho} \circ T_{\varphi}$. Этот классический результат Данжуа без труда переносится на случай гомеоморфизмов окружности, удовлетворяющих условиям теоремы 1 . Вопрос о гладкости сопрягающего гомеоморфизма $T_{\varphi}$ тесно связан с существованием абсолютно непрерывной инвариантной меры для $T_{f}$. При этом инвариантная мера $\mu$ для $T_{f}$ является абсолютно непрерывной относительно меры Лебега $\lambda$ тогда и только тогда, когда сопряжение $T_{\varphi}$ задается абсолютно непрерывной функцией. 
Гомеоморфизмы окружности с одной точкой излома впервые были изучены в работе Е. Б. Вул и К. М. Ханина [3]. С одной стороны, как и в случае гладких диффеоморфизмов, ренормализация таких гомеоморфизмов ведет себя достаточно просто. В перенормированных координатах итерации отображения $T_{f}$ асимптотически близки к дробно-линейным преобразованиям, зависящим только от двух параметров [3]. С другой стороны, наличие одной точки излома существенно меняет ситуацию. А именно, если гомеоморфизм является гладким всюду за исключением точки излома и принадлежит классу $C^{2+\varepsilon}$ при некотором $\varepsilon>0$, то в случае иррационального числа вращения инвариантная мера сингулярна относительно меры Лебега [4].

Рассмотрим гомеоморфизм $T_{f}$, удовлетворяющий условиям (a)-(e). Дополнительно предположим, что все точки излома лежат на одной траектории гомеоморфизма $T_{f}$, т.е. существует точка $x_{0} \in S^{1}$ такая, что $z_{j}=T_{f}^{p_{j}} x_{0}, p_{j} \in \mathbb{Z}_{+}$.

Теорема 2. Предположим, что $\prod_{j=0}^{m} c_{j} \neq 1$ и число вращения $\rho=\rho\left(T_{f}\right)$ иррационально. Тогда инвариантная мера $\mu$ сингулярна относительно меры Лебега $\lambda$.

ОПредЕлЕниЕ 2 . Две меры $\nu_{1}$ и $\nu_{2}$ на одной $\sigma$-алгебре называются $L_{2}$-эквивалентными, если существуют функции $\varphi_{1} \in L_{2}\left(\nu_{1}\right), \varphi_{2} \in L_{2}\left(\nu_{2}\right)$ такие, что $\nu_{1}=\varphi_{2} \nu_{2}$ и $\nu_{2}=\varphi_{1} \nu_{1}$.

Рассмотрим разложение иррационального числа вращения $\rho \in(0,1)$ в непрерывную дробь: $\rho=\left[k_{1}, k_{2}, \ldots, k_{n}, \ldots\right]$.

ОПРЕдЕЛЕниЕ 3. Иррациональное число $\rho \in(0,1)$ называется числом "ограниченного типа", если последовательность $\left\{k_{n}, n=\overline{1, \infty}\right\}$ ограничена.

Теорема 3. Пусть число вращения $\rho=\rho\left(T_{f}\right)$ является иррациональным числом ограниченного типа $и \prod_{j=1}^{m} c_{j}=1$. Тогда инвариантная мера $L_{2}$-эквивалентна мере Лебега.

ЗАмечАния 1. 1. Существенно, что теоремы 2 и 3 доказываются для отображений низкой гладкости, т.е. удовлетворяющих условию (е). В случае, когда $T_{f}$ принадлежит классу $C^{2+\varepsilon}$ вне точек излома, соответствующие результаты могут быть доказаны проще. В этом случае утверждение теоремы 2 является простым обобщением результата работы [4], а теорема 3 следует из [5], где близкий результат доказан для типичных иррациональных чисел вращения. Довольно ограничительное условие на число вращений (числа ограниченного типа) связано с работой И. Кацнельсона и Д. Орнстейна [6], многие идеи из которой существенно используются при доказательстве теоремы 3. Можно сказать, что теорема 3 является развитием теории Кацнельсона и Орнстейна на случай гомеоморфизмов окружности с изломами.

2. Интересным представляется вопрос об инвариантных мерах в общем случае, т.е. когда точки излома не лежат на одной траектории. В этом случае имеются основания предполагать, что инвариантная мера всегда сингулярна относительно меры Лебега.

Автор благодарен А. А. Джалилову и К. М. Ханину за ценные обсуждения. Существенная часть работы была выполнена во время визита автора в Эдинбург, которой стал возможен благодаря финансовой поддержке гранта от Королевского Общества Великобритании.

\section{Список литературы}

[1] И. П. Корнфельд, Я.Г. Синай, С. В. Фомин, Эргодическая теория, Наука, М., 1980. [2] A. Denjoy, J. Math. Pures Appl. (9), 11 (1932), 333-375. [3] K. M. Khanin, E. B. Vul, Adv. Soviet Math., 3 (1991), 57-98. [4] А. А. Джалилов, К. М. Ханин, Функи. анализ и его прилож., 32:3 (1998), 11-21. [5] А. А. Джалилов, ТMФ, 120:2 (1999), 179-192. [6] Y. Katznelson, D. Ornstein, Ergodic Theory Dynam. Systems, 9:4 (1989), 681-690.

Х. А. Ахадкулов (Kh. А. Akhadkulov) Самаркандский государственный университет им. Алишера Навои
Представлено Д. В. Аносовым Принято редколлегией 01.08 .2006 